\title{
BLACK GOLD - LIKE SERS-ACTIVE METAL-ORGANIC FRAMEWORKS
}

\author{
Daria CECICO, Olga GUSELNIKOVA, Pavel POSTNIKOV, Vaclav SVORCIK, Oleksiy LYUTAKOV \\ UCT Prague- University of Chemistry and Technology, Prague, Czech Republic, EU, \\ cecicod@vscht.cz
}

https://doi.org/10.37904/nanocon.2019.8533

\begin{abstract}
We propose a novel black gold-like structure on the base of plasmonic nanostructures incorporated in the MOFs cavities for potential application in solar energy and photochemistry. Mean hybrid structure was prepared by sputtering of gold on the MOFs decorated substrates. Since the size of gold nanostructures, created during the deposition procedure is smaller than the size of pores in MOFs, the gold atoms filled the 3D MOFs structure and formed the nanostructures with wide distribution of sizes and shapes. The surface and bulk properties of created hybrid materials were carefully characterized. In particular, the presence and distribution of gold nanoparticles was confirmed by SEM-EDX and TEM measurements. Plasmonic properties were characterized by UV-Vis spectroscopy and SERS measurements using R6G as model analyte. Our results suggest that created hybrid material is a potential candidate for application in the field of black gold utilization, for example affective light absorber and convertor.
\end{abstract}

Keywords: MOFs, gold nanoparticles, hybrid material, plasmonics, photochemistry

\section{INTRODUCTION}

Metal-organic frameworks (MOFs) are crystalline porous solids composed of a three-dimensional network of metal ions held in place by multidentate organic molecules [1,2]. The spatial organization of these structural units leads to a system of channels and cavities in the nanometer length scale. The metal ions form nodes that connect the arms of the linking molecules together to form a repeating cage-like structure. Thanks to this hollow structure, MOFs have an extremely large inner surface and thus MOFs are very promising candidates for many applications: gases or liquids separation, purification and storage, electrochemical energy conversion and storage, catalysis and sensor technologies as well as potential precursors for the construction of inorganic functional materials.

Especially interesting is the possibility to combine plasmon active nanostructures with MOFs, since obtained hybrid materials potentially will have a range of advanced properties resulted from vast inner surface of MOFs and broad plasmon resonance with wide spectrum of light absorption. [3-5]. Plasmon is the collective oscillation of electrons, excited by the external electromagnetic wave on the metal-dielectric boundary and could be considered as an extremely effective lens, because it is featured by the strongly localized evanescent wave. This plasmon-based lens focuses light beyond the diffraction limit. Unique plasmon's properties have been extensively studied for Raman spectroscopy, plasmonic devices, and chemical transformation processes [611].

Because of the huge interest in the development of materials for harvesting solar energy, it is very important to achieve a perfect absorber, so the plasmonic hybrids are the materials of interest. Sophistically created complex plasmonic structures, composed the self-assembled nanoparticles would induce the nearfield coupling of surface-plasmons between neighboring nanoparticles, with corresponded increase of light absorption efficiency [12-14].

In this contribution we propose a new designed of material combining MOFs porous structure with Au nanoclusters trapped inside it. The resulting material mimics the properties of a porous gold film with a nonreflective surface. We suggest that this hybrid would behave as a black gold, which is a material with high 
absorbance properties. [16-17]. However, manufacturing of Au-black films is usually expensive and timeconsuming; our method is very simple and easily reproducible even in the smallest lab.

\section{RESULTS AND DISCUSSION}

The schematic representation of proposed experimental concept is presented in Figure 1. First, the powder of MOF- 5 was synthesized in solvothermal conditions using zinc nitrate hexahydrate, terephthalic acid and N, $\mathrm{N}$-Dimethylformamide as precursor materials. Then the duct tape was put on the glass and the MOF- 5 powder was manually deposited on the tape. The samples were always blow dried to remove the excessive MOF-5 powder. To make sure that all the samples are equal, the same size of glass and tape were always used. In the next step the Au sputtering was applied with the aim to create Au nanostructures in MOF-5 pores. Different sputtering times were used, to modify the amount of Au nanostructures within the MOF- 5 powder.

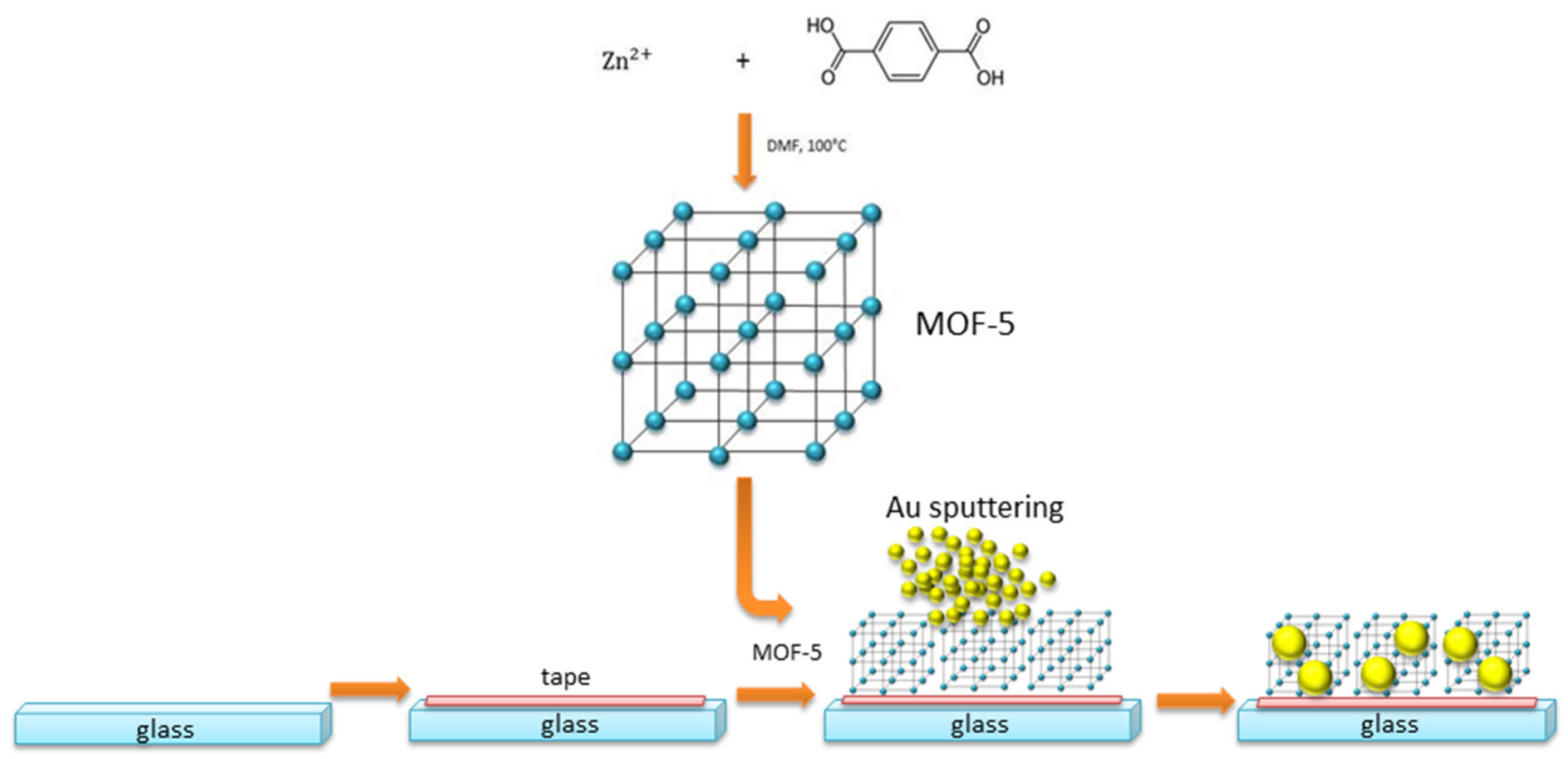

Figure 1 Schematic representation of sample preparation

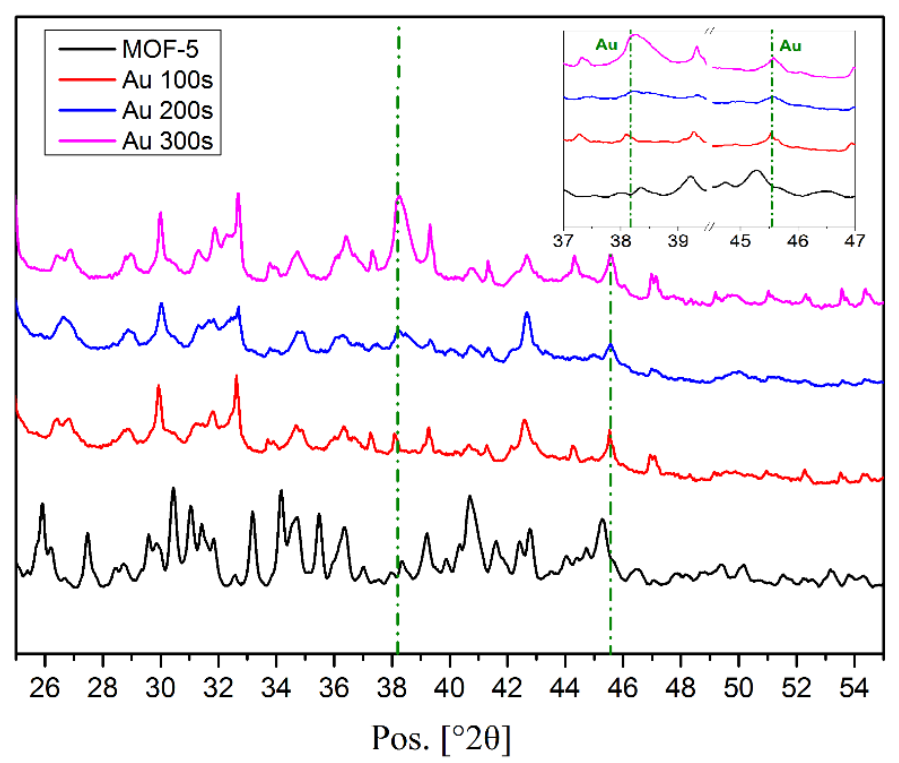

Figure 2 XRD spectra of MOF-5 powder sputtered with Au for 100, 200 and 300 seconds 
To confirm the composition of the obtained MOF- 5 structure the XRD method was applied. Obtained results are presented in the Figure 2. Comparison with previously published XRD graphs indicates the presence of main MOF-5 characteristic peaks [18]. The Figure 2 also shows the XRD results obtained after the deposition of Au on MOF-5 powder (deposition time: 100, 200 and 300 seconds). Appearance of several additional peaks indicates the formation of crystalline Au phase. Affiliation of mean peaks ( 38 and $46{ }^{\circ} 2 \theta$ ) shows that the Au forms the polycrystalline structures.

The size and its distribution of deposited Au clusters were measured by TEM. Results are presented in the Figure 3 as a function of Au amount depending on sputtering time.
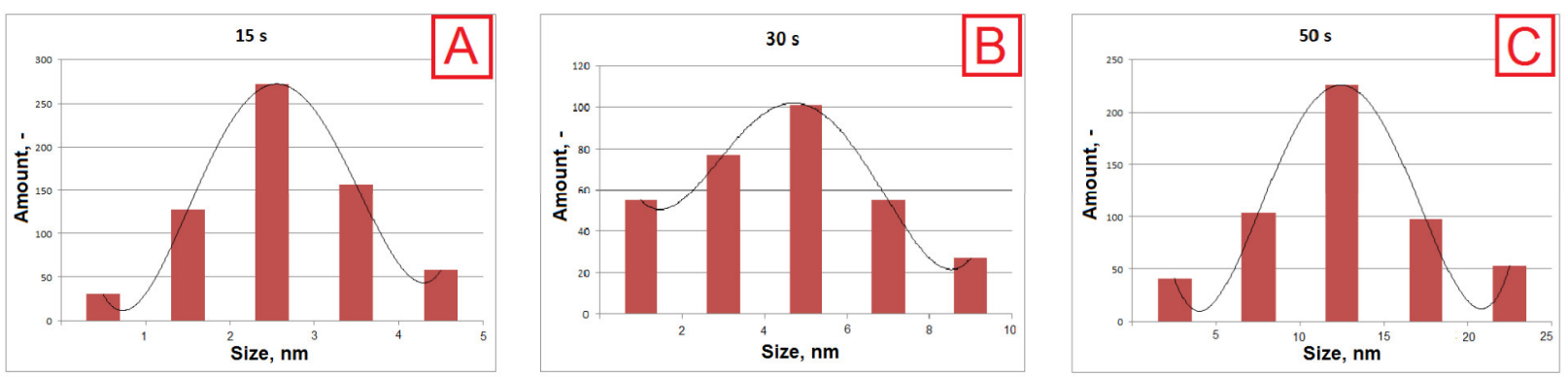

Figure 3 Size-amount distribution of Au NPs in MOF-5 after (A) 15 seconds sputtering, (B) 30 second sputtering, (C) 50 seconds sputtering

Using TEM the approximated size distribution of Au nanostructures was evaluated (Figure 4). As is evident from the scans the shorter time of deposition (15 seconds) leads to the formation of Au clusters with the sizes from $1 \mathrm{~nm}$ to $5 \mathrm{~nm}$. Increasing of sputtering time to 30 seconds results in the shift Au clusters size increase (2-10 nm). The further increase of deposition time (up to 50 seconds) leads to formation of even higher nanoparticles with wider distribution of size. Finally, after the Au deposition for 100 seconds the random interpenetrated Au structures is formed. From random distribution of Au clusters size, shape and gaps between them, the excitation of broad plasmon resonance can be expected (i.e. absorption through the wide wavelength range), which is mainly typical for black gold.
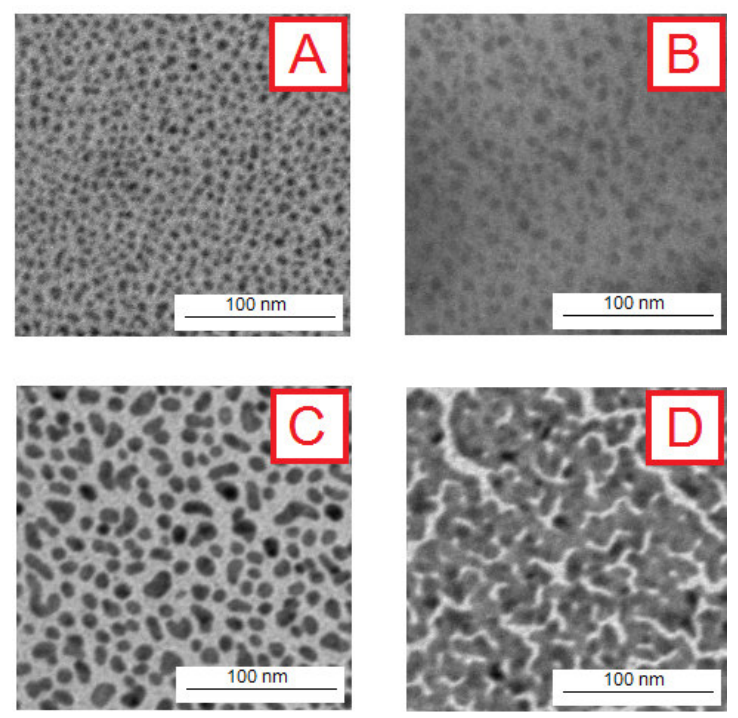

Figure 4 TEM images of MOF-5 sputtered with $\mathrm{Au}$ for (A) 15 seconds, (B) 30 seconds, (C) 50 seconds, (D) 100 seconds
100
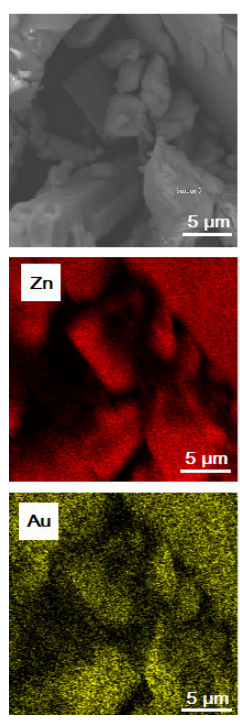

200
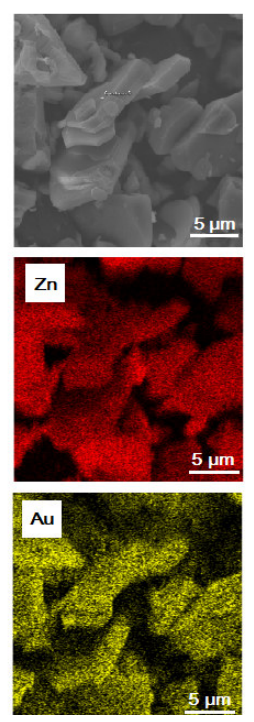

300
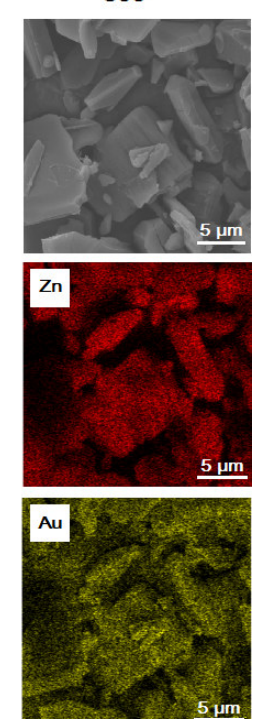

Figure 5 SEM-EDX images of MOF-5 sputtered for 100,200 and 300 seconds 
SEM-EDX results (Figure 5) allow determining the spatial position of the MOF-5 microcrystals as well as the presence of the Au nanoclusters inside it. In particular, the comparison of EDX elements mapping (especial attention deserves the distribution of $\mathrm{Zn}$ and $\mathrm{Au}$ ) with SEM images confirms that the Au nanostructures were trapped inside the MOF-5 structure. As it is seen from the Figure 5 the mappings on $\mathrm{Zn}$ and Au are always the same. Since $\mathrm{Zn}$ is the main characteristic part of the MOF- 5 structure, these results indicate that during the deposition Au nanostructures fall in the cage-like structure of MOF-5.

Optical properties were characterized by UV-Vis spectroscopy. Results are presented in Figure 6 . These measurements show the increase of the absorbance with the sputtering time. It is clearly seen, that MOF- 5 powder itself does not absorb the light effectively. After the Au deposition overall absorption in the 500-900 nm wavelengths range increases. It is important to mention, that there is the linear dependency between the time of sputtering and the power of absorption. Since the refraction coefficient was closed to zero, observed differences in UV-Vis spectra should be attributed solely to intrinsic absorption increase. So, we can confirm our assumption that MOFs sputtered with Au behaves as Au-black.

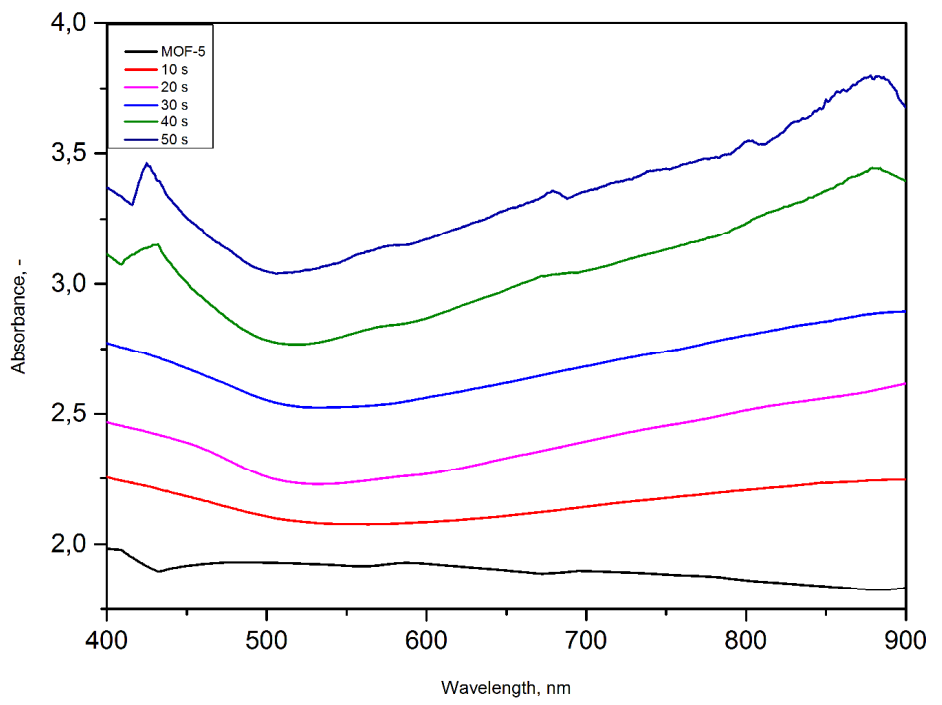

Figure 6 UV-VIS spectra of MOF-5 sputtered for 10, 20, 30, 40 and 50 seconds
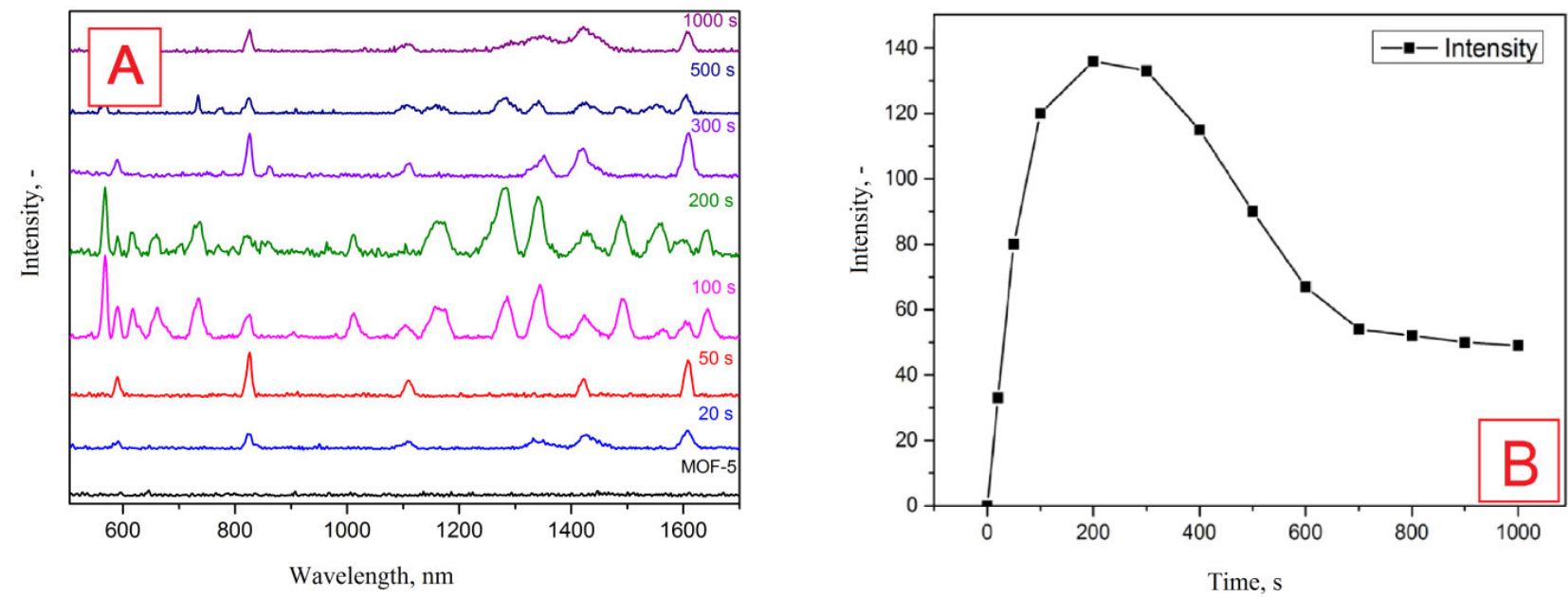

Figure 7 (A)Raman spectra of MOF-5 sputtered for 0, 20, 50, 100, 200, 300, 500, 1000 seconds with R6G, (B) Dependence of the Raman spectrum signal intensity on the Au sputtering time 
Finally, the plasmon activity was checked through the SERS measurement. For this goal our samples were sputtered for 20,50,100, 200,300, 500 and 1000 seconds and then the Raman spectra were measured using R6G as model SERS analyte. R6G was drop-deposited on samples surface (and penetrates in MOF-5/Au pores) and then dried under ambient conditions. After the comparison of the Raman spectra of MOF-5 powder without Au deposited with other spectra, it is seen that the R6G Raman response is significantly enhanced by Au clusters (which is the typical SERS phenomenon). The dependency of SERS enhancement (estimated as characteristic peak intensity increase $-1346 \mathrm{~nm}$ ) on the Au sputtering time is not linear. The mean intensity growths until the 200 seconds of sputtering, then the intensity decreases and slowly becomes constant. So, it can be concluded that the more effective plasmon excitation occurs in the MOF- 5 structures, containing the 200 sec. sputtered Au clusters.

\section{CONCLUSION}

To sum up, we prepared and tested a novel type of a black-gold like structure. Our analyses confirmed the expectations about the properties of MOF- 5 sputtered with Au. According to the XRD, TEM and SEM-EDX Au nanoclusters are clearly formed and stay trapped inside the MOF- 5 structure. Since MOFs is a 3D crystalline porous material, by being sputtered with Au nanoparticles, plasmon active hybrid material is created. UV-Vis demonstrated the ability of our samples to effectively absorb the light in a Vis and near IR wavelengths range. The SERS measurements confirm the plasmon activity of created material. It was also established that the optimum time of sputtering is 200 seconds; this time gives the best plasmon excitation. Proposed black goldlike SERS-active metal-organic frameworks can found their application in in solar energy conversion and photochemistry.

\section{ACKNOWLEDGEMENTS}

This work was financially supported by the Internal Grant Agency UCT № 1260881906

\section{REFERENCES}

[1] M. EDDAOUDI, D.B. MOLER, H.L. LI, B.L. CHEN, T.M. REINEKE, M. O'KEEFFE, O.M. YAGHI, Acc. Chem. Res. 34, 2001, 319.

[2] S.L. JAMES, Chem. Soc. Rev. 32, 2003, 276.

[3] N.L. ROSI, J. ECKERT, M. EDDAOUDI, D.T. VODAK, J. KIM, M. O'KEEFFE, O.M. YAGHI, Science 300, 2003, 1127.

[4] G. FÉREY, C. MELLOT-DRAZNIEKS, C. SERRER, F. MILLANGE, J. DUTOUR, S. SURBLI, J. MARGIOLAKI, Science 309, 2005, 2040.

[5] O. GUSELNIKOVA, P. POSTNIKOV, R. ELASHNIKOV, E. MILIUTINA, V. SVORCIK, LYUTAKOV, O. Anal. Chim. Acta, 1068, 201970.

[6] O. GUSELNIKOVA, P. POSTNIKOV, M. M. CHEHIMI, Y. KALACHYOVAA, V. SVORCIK, O. LYUTAKOV, Langmuir, 35, 2019, 2023.

[7] F. LLABResiXAMENA, A. ABAD, A. CORMA, H. GARCIA, J. Catalysis, 250, 2007, pp. 294-298

[8] W. L. BARNES, A. DEREUX, T. W. EBBESEN, Nature 424, 2003, 824.

[9] J. ŠVANDA, Y. KALACHYOVA, P. SLEPICKA, V. SVORCIK, O. LYUTAKOV, ACS Appl. Mater. Interfaces, 8, 2016, 225.

[10] M. L. BRONGERSMA, N. J. HALAS, P. NORDLANDER, Nat. Nanotechnol. 10, 2015, 25.

[11] A. J. HAES, R. P. VAN DUYNE, Anal. Bioanal. Chem. 379, 2004, 920.

[12] K. A. WILLETS, R. P. VAN DUYNE, ANNU. Rev. Phys. Chem. 58, 2007, 267. 
[13] A. CHEN, A. E. DEPRINCE, A. DEMORTIÈRE, A. JOSHI-IMRE, E. V. SHEVCHENKO, S. K. GRAY, U. WELP, V. K. VLASKO-VLASOV, Small 7, 2011, 2365;

[14] D. LIU, F. ZHOU, C. LI, T. ZHANG, H. ZHANG, W. CAI, L. YUE GDCh, 54, 2015, 9596.

[15] X. LIU, T. TYLER, T. STARR, A. F. STARR, N. M. JOKERST, W. PADILLA, Phys. Rev. Lett, $107,2011,045901$

[16] H. TAO, C. M. BINGHAM, A. C. STRIKWERDA, D. PILON, D. SHREKENHAMER, N. I. LANDY, K. FAN, X. ZHANG, W. J. PADILLA R. D. AVERITT, Phys. Rev. B 78, 2008, 241103.

[17] T. V. TEPERIK, F. J. G. ABAJO, A. G. BORISOV, M. ABDELSALAM, P. N. BARTLETT, Y. SUGAWARA, J. J. BAUMBERG Nature Photon. 2, 2008, 299.

[18] B. CHEN, X. WANG, Q. J. ZHANG, X. XI, J. CAI, H. QI, S. SHI, J. WANG, D. YUANA M. FANG, J. Mater. Chem., $20,2010,3758$. 